ETHZ-IPP PR-96-01

21 March, 1996

\title{
Neutral Current Interference in the TeV Region; the Experimental Sensitivity at the LHC
}

\author{
Michael Dittmar \\ Institute for Particle Physics (IPP), ETH Zürich, \\ CH-8093 Zürich, Switzerland
}

\begin{abstract}
The possibilities to measure lepton forward-backward asymmetries at the LHC in the reaction $\operatorname{pp}\left(q_{i} \overline{q_{i}}\right) \rightarrow \ell^{+} \ell^{-}$are studied for dilepton events with masses above $400 \mathrm{GeV}$. It is shown that such measurements allow accurate tests of the neutral current interference structure up to about $2 \mathrm{TeV}$ center of mass energies. The sensitivity of asymmetries at the LHC to new physics is demonstrated within the context of quark compositeness and exotic $Z^{\prime}$ scenarios.
\end{abstract}




\section{Introduction}

Measurements of forward-backward charge asymmetries, $\mathrm{A}_{\mathrm{fb}}$, are traditionally a domain of $\mathrm{e}^{+} \mathrm{e}^{-}$collider experiments. Evidence for the $\gamma-\mathrm{Z}$ interference came from the observation of the non zero value of $\mathrm{A}_{\mathrm{fb}}$ in the reaction $\mathrm{e}^{+} \mathrm{e}^{-} \rightarrow \mu^{+} \mu^{-}$at PETRA around 1982/1983 [1]. Furthermore, $\mathrm{A}_{\mathrm{fb}}$ measurements with quarks and leptons at the $\mathrm{Z}$ peak provide precise $\sin ^{2} \theta_{\mathrm{w}}$ determinations [2]. The sensitivity of $\mathrm{A}_{\mathrm{fb}}$ measurements to new physics like additional $\mathrm{Z}^{\prime}$ bosons or compositeness has been shown in many details for future high energy $\mathrm{e}^{+} \mathrm{e}^{-}$collider experiments [3]. Lepton asymmetries in the reaction $\mathrm{p}-\overline{\mathrm{p}} \rightarrow \mathrm{e}^{+} \mathrm{e}^{-}\left(\mu^{+} \mu^{-}\right)$can be defined with respect to the proton direction. Such asymmetry measurements, [4], are currently less precise than the corresponding $\mathrm{e}^{+} \mathrm{e}^{-}$results and have so far only been performed close to the $\mathrm{Z}$ resonance.

High mass dilepton events in $\mathrm{p}-\mathrm{p}$ collisions originate from the annihilation of valence quarks with sea-antiquarks or from the annihilation of sea-quarks with sea-antiquarks. As the valence quarks have on average a much larger momentum than the sea-antiquarks, the boost direction of the dilepton system approximates the quark direction. A lepton asymmetry can thus be expected with respect to the boost direction. In contrast, dilepton events which originate from the annihilation of quark pairs from the sea must be symmetric.

The sensitivity of $\mathrm{A}_{\mathrm{fb}}$ measurements to $\mathrm{Z}^{\prime}$ bosons at future multi $\mathrm{TeV} \mathrm{p}-\mathrm{p}$ and $\mathrm{p}-\overline{\mathrm{p}}$ has been discussed by several Authors [5]. In particular P. Langacker et al. have studied the sensitivity of on-resonance asymmetries to the couplings of hypothetical new bosons at future multi TeV hadron colliders. Such on-resonance asymmetry measurements at the LHC were simulated in detail for the 1990 workshop in Aachen [6] and also for the design studies of ATLAS [7] and CMS [8]. The possibility of an asymmetry measurement at the Z peak with a dedicated LHC experiment has also been discussed [9].

However, asymmetries from continuum dilepton events at very high energy pp colliders as an additional tool to study the neutral current interference have so far only been studied theoretically. J. Rosner has investigated the sensitivity of lepton asymmetries with on-resonance, off-resonance and continuum dilepton events at the SSC. He concluded that the combination of all these measurements should provide the best sensitivity to new physics in neutral current reactions [10].

With the more clearly defined experimental capabilites of the LHC experiments ATLAS [7]

and CMS [8] and an expected integrated yearly luminosity of up to $100 \mathrm{fb}^{-1}$ per experiment it becomes interesting to perform a simulation of such an asymmetry measurement at the LHC. Such an analysis and its sensitivity to new physics is described in the following.

\section{Asymmetries in reactions with quark and lepton pairs}

The reactions $\operatorname{pp}\left(q_{i} \overline{q_{i}}\right) \rightarrow e^{+} e^{-}$and $\operatorname{pp}\left(q_{i} \overline{q_{i}}\right) \rightarrow \mu^{+} \mu^{-}$, as well as the inverse reaction $e^{+} e^{-} \rightarrow$ $q_{i} \overline{q_{i}}$ are described by the exchange of neutral vector bosons. Within the Standard Model the couplings of the photon and the $\mathrm{Z}$ to quarks and leptons are known and precise calculations for the interference between the photon and the $\mathrm{Z}$ exist. The $\gamma-\mathrm{Z}$ interference results in large 
forward-backward asymmetries for center of mass energies well above the $\mathrm{Z}$ peak. For center of mass energies above $250 \mathrm{GeV}$, essentially constant asymmetries of about $61 \%$ are expected for the above reactions with up-type and down-type quarks. New phenomena in the TeV range, like $\mathrm{Z}^{\prime}$ bosons [5] or contact interactions between quarks and lepton due to compositeness [11] might considerably modify this picture. Fermion asymmetry measurements at high center of mass energies are consequently an excellent tool to search for and perhaps study such new phenomena.

Asymmetries with quarks and leptons in the final state up to $m_{\ell \ell}(=\sqrt{s}) \approx 200 \mathrm{GeV}$ will be measured in detail at LEP II and the region of perhaps up to $m_{\ell \ell} \approx 500 \mathrm{GeV}$ will be investigated at the upgraded TEVATRON p $\overline{\mathrm{p}}$ collider 12 . To exploit the higher energies at the LHC, we concentrate in this study on high mass dilepton events $\left(m_{\ell \ell}>400 \mathrm{GeV}\right)$.

In pp collisions, unlike in $\mathrm{e}^{+} \mathrm{e}^{-}$collider experiments, the center of mass frame is different from the laboratory frame. However, the four momenta of the dilepton system are measured and one can calculate the lepton (electron or muon) angle $\theta^{*}$ in the dilepton center of mass frame. The lepton asymmetry $\mathrm{A}_{\mathrm{FB}}^{\ell}$ is defined from the angular distribution $\cos \theta^{*}$ with respect to the quark direction and can be obtained best with an unbinned maximum likelihood fit to the $\cos \theta^{*}$ distribution given by:

$$
\frac{d \sigma}{d \cos \theta^{*}} \propto 3 / 8\left(1+\cos ^{2} \theta^{*}\right)+\mathrm{A}_{F B}^{\ell} \cos \theta^{*}
$$

To simulate an asymmetry measurement with dilepton events at the LHC (with $14 \mathrm{TeV}$ pp collisions) the PYTHIA Monte Carlo |13 is used. The PYTHIA dilepton event generators allow to simulate the Standard Model predictions with a photon and a Z exchange as well as modifications due to compositeness [14 or the possibilities of the additional exchange of a $Z^{\prime}$ including the interference between $\gamma, \mathrm{Z}$ and $\mathrm{Z}^{\prime}$ [15].

For the Standard Model simulation of dilepton events with a mass larger than $400 \mathrm{GeV}$, one finds that about $70 \%$ originate from the annihilation of $u \bar{u}$ quarks, $21 \%$ from $d \bar{d}$ quarks and about $9 \%$ are from the annihilation of $s \bar{s}, c \bar{c}$ and $b \bar{b}$ sea-quarks. Uncertainties from the quark flavour composition are not important for comparisons of a measurement with the Standard Model as essentially identical lepton asymmetries are predicted for the annihilation of $u \bar{u}$ and $d \bar{d}$ quark pairs and dilepton masses above $400 \mathrm{GeV}$. However, at a pp collider, observable asymmetries must come from those dilepton events which originate from the annihilation of a valence quark ( $\mathrm{u}$ and $\mathrm{d}$ quarks) and the corresponding sea-antiquark. The fraction of events which will show measurable asymmetries depends therefore on the mass and on the boost of the dilepton system.

As a first step of the simulation, the charge asymmetries are determined with respect to the quark direction taken from the generator. Using this approach lepton asymmetries of $\approx 61 \%$ are obtained from the fit to the $\cos \theta^{*}$ distribution of dilepton events and the Standard Model simulation. The asymmetry is essentially independent of the mass and rapidity of the lepton system. If instead a random assignment for the quark direction is used, the angular distribution of the leptons is well described by a $1+\cos ^{2} \theta^{*}$ function with an asymmetry of zero.

As the original quark direction is not known in pp collisions one has to extract it from the kinematics of the dilepton system. For this analysis, the quark direction is taken from the boost 
direction of the dilepton system with respect to the beam axis (the z-axis). The correctness of this assignment is studied as a function of the dilepton rapidity y $=1 / 2 \times \ln \left[\left(E+p_{z}\right) /\left(E-p_{z}\right)\right]$. The rapidity distribution for dilepton events with masses above $400 \mathrm{GeV}$ is shown in Figure 1a for all dilepton events and for the subsample of events where the sign of the boost direction and the quark direction agree. As shown in Figure 1b, the fraction of events with a correctly assigned quark direction increases as a function of the rapidity. For small rapidities $(|y|<0.2)$ essentially all dilepton events originate from the annihilation of sea-quarks with sea-antiquarks and an almost random assignment is made for the quark direction. The fraction of events with a correctly assigned quark direction increases to more than $80 \%$ at a rapidity of one.
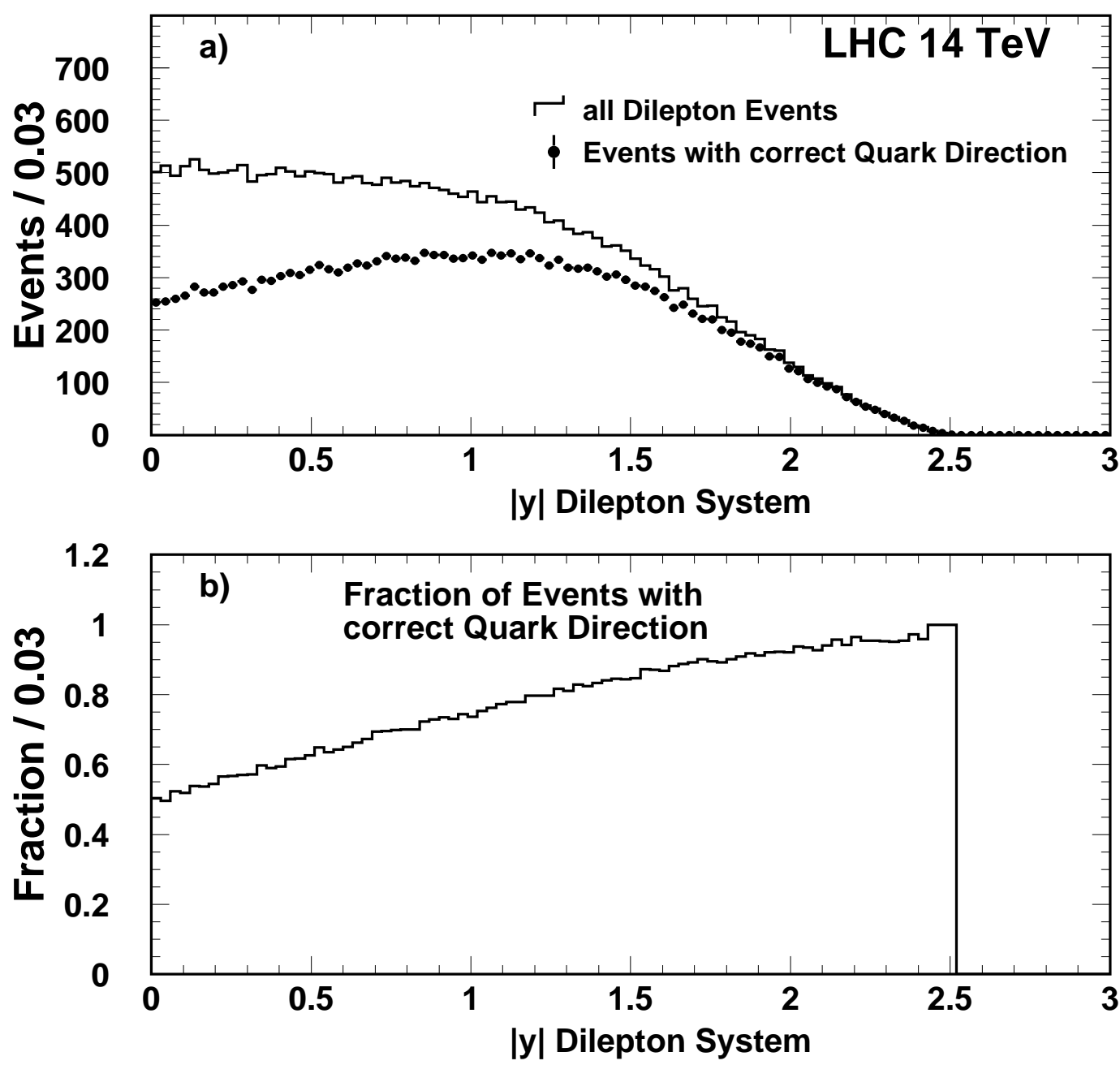

Figure 1: Rapidity $|y|$ distribution of the dilepton system with respect to the beam direction for all events and the subsample where the sign of the rapidity agrees with the quark direction (a). The fraction of events with a correctly assigned quark direction is shown in (b). 
Uncertainties for the quark direction assignment and from the fraction of events which originate from the annihilation of sea-quark pairs have been studied with a variety of structure functions [16] as implemented in PYTHIA. From this study we find that for dilepton masses above $400 \mathrm{GeV}$ these uncertainties are essentially negligible compared to the achievable statistical precisions of the $\mathrm{A}_{\mathrm{fb}}^{\ell}$ measurements. However, for dilepton masses below $200 \mathrm{GeV}$, the uncertainties are more important for asymmetry measurements and require special attention. As this center of mass energy range will already be covered by LEP II, we concentrate in the following only on dilepton events with high mass.

\section{Simulation of an Asymmetry Measurement}

According to the design characteristics of ATLAS [7] and CMS [8] high identification efficiency for isolated electrons and muons with excellent energy and momentum resolutions are expected down to pseudorapidities of $|\eta|<2.5$ and for the highest LHC luminosities $\left(10^{34} \mathrm{~cm}^{-2} \mathrm{sec}^{-1}\right)$. For example, using the beam spot constraint, the momentum resolution for $1 \mathrm{TeV}$ muons and electrons should be better than $10 \%$ and no charge confusion is expected up to much higher momenta. With this accuracy, dilepton mass resolutions of better than $5-10 \%$ for dimuon events and about $1-2 \%$ for dielectron events can be expected for masses of about $1 \mathrm{TeV}$.

For this study, PYTHIA events of the type $\mathrm{pp} \rightarrow e^{+} e^{-}(\gamma)$ and $\mathrm{pp} \rightarrow \mu^{+} \mu^{-}(\gamma)$ are simulated at a center of mass energy of $14 \mathrm{TeV}$. The produced leptons are in general isolated and are essentially back to back in the plane transverse to the beam direction. Dilepton events, either dielectron or dimuon events, are accepted if the following criteria are fulfilled:

- The minimum $p_{t}$ of each charged lepton should be larger than $20 \mathrm{GeV}$.

- The pseudorapidity $|\eta|$ of each lepton should be smaller than 2.5 .

- The two leptons must have opposite charge.

- The two leptons should be back to back in the plane transverse to the beam direction with an opening angle between the two leptons of more than $160^{\circ}$.

- The dilepton mass has to be larger than $400 \mathrm{GeV}$.

Especially for dilepton pairs with masses in the $\mathrm{TeV}$ region these criteria seem to be sufficient to select essentially background free events. However, if required by backgrounds, isolation criteria for electrons and muons and veto criteria against events which have a large jet activity, can be applied without a significant loss in statistics.

For an integrated luminosity of $100 \mathrm{fb}^{-1}$ about 28k dilepton events with a mass above $400 \mathrm{GeV}$ are accepted with these criteria. Table 1 shows the number of expected events for different mass intervals. For a real experiment the number of events will be further reduced by the imperfect geometrical detector coverage and lepton detection efficiencies $\epsilon$. The number of events, given in Table 1 should be multiplied by $\epsilon^{2}$ and the estimated asymmetry errors will 
increase by roughly $1 / \epsilon$. Accurate efficiency estimates are difficult to make, but values of $90 \%$ and more have been used for other simulations [17].

As discussed in section 2, the lepton asymmetry is obtained from the $\cos \theta^{*}$ distribution in the dilepton center of mass frame. The $\cos \theta^{*}$ distribution for dilepton masses between 0.75 $\mathrm{TeV}$ and $1.25 \mathrm{TeV}$ and an absolute rapidity of more than 0.8 is shown in Figure 2a before and after the selection criteria are applied. As expected, the used lepton selection criteria effect only large $\left|\cos \theta^{*}\right|$ values.
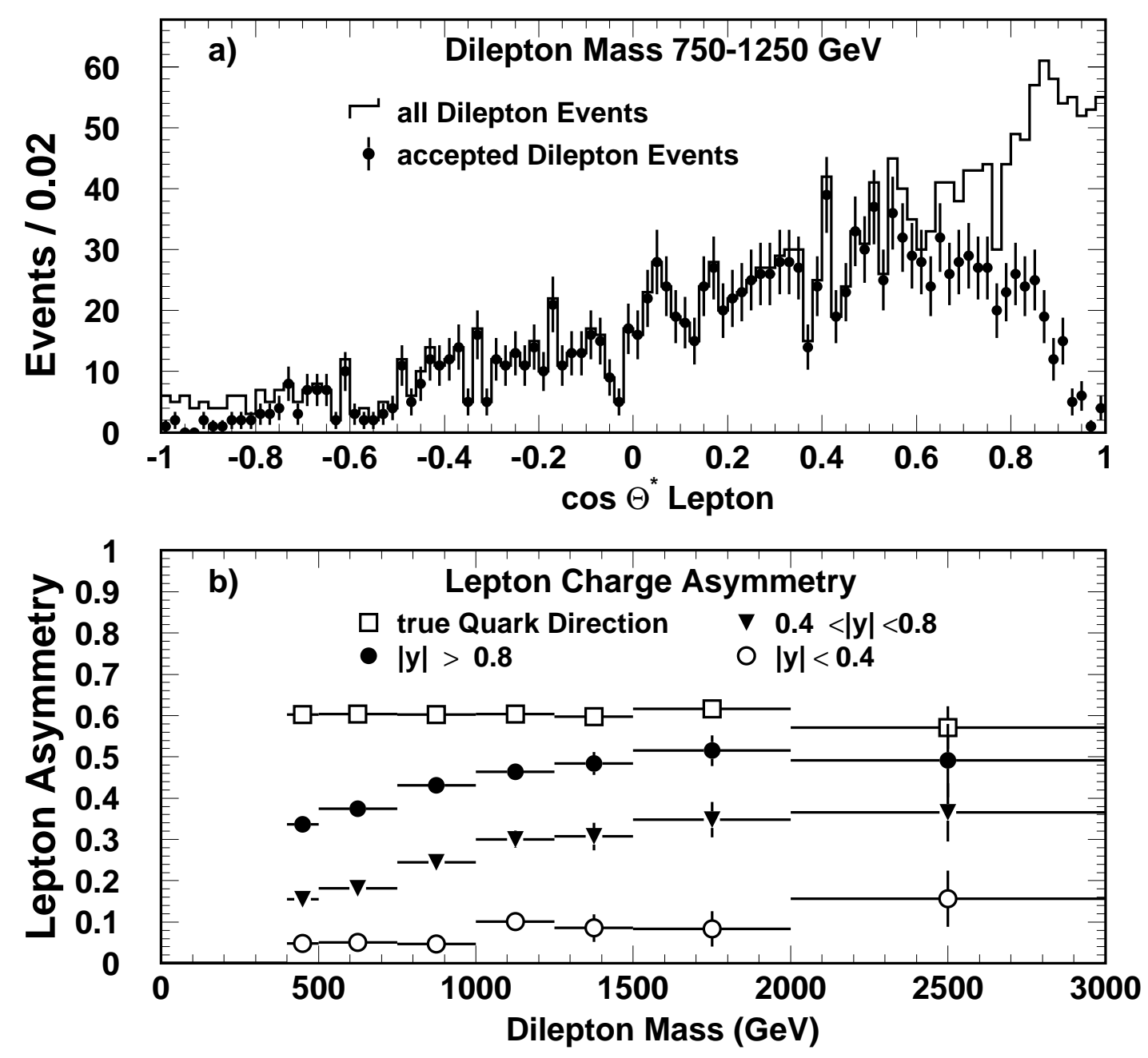

Figure 2: (a) The Standard Model predictions for the $\cos \theta^{*}$ distribution of the negatively charged lepton with and without acceptance criteria for a mass of the dilepton system in the interval $0.75-1.25 \mathrm{TeV}$ and an absolute rapidity of the dilepton system larger than 0.8 . Asymmetries for different dilepton mass and rapidity intervals are shown in (b). 
Lepton asymmetries are now determined from the $\cos \theta^{*}$ distribution of the negatively charged lepton with respect to the boost direction of the dilepton system along the $\mathrm{z}$ axis. For various dilepton mass and rapidity intervals, the $\cos \theta^{*}$ distribution is fitted using the unbinned maximum likelihood method. These lepton asymmetries are shown in Figure $2 \mathrm{~b}$ as a function of the dilepton mass (using about 10 times the integrated luminosity expected per experiment). Large and statistically significant asymmetries are found for large dilepton rapidities up to masses of about $2 \mathrm{TeV}$.

The extracted asymmetries are smaller than the ones expected from the Standard Model and depend on the mass and rapidity of the dilepton system. This difference comes from two sources, the contributions from events which originate from the annihilation of sea-quarks which must have zero asymmetry and from the cases where the valence quark carries a smaller momentum than the sea-antiquark. Both contributions reduce the asymmetries and can be considered as random background. This background is especially important for low dilepton masses. Possible uncertainties from these backgrounds were studied and found to be much smaller than the obtainable statistical errors from an integrated luminosity of $100 \mathrm{fb}^{-1}$. The expected Standard Model asymmetries, uncorrected for backgrounds, with statistical errors assuming an integrated luminosity of $100 \mathrm{fb}^{-1}$ are given in Table 1.

\begin{tabular}{|c|c|c|c|c|}
\hline $\operatorname{mass}\left(\ell^{+} \ell^{-}\right)$ & Number of Events & \multicolumn{3}{|c|}{$\mathrm{A}_{\mathrm{FB}}^{\ell}$} \\
\hline$[\mathrm{TeV}]$ & $\left(\right.$ for $\left.100 \mathrm{fb}^{-1}\right)$ & $|y|<0.4$ & $0.4<|y|<0.8$ & $|y|>0.8$ \\
\hline $0.40-0.50$ & 14100 & $0.05 \pm 0.02$ & $0.16 \pm 0.02$ & $0.34 \pm 0.01$ \\
$0.50-0.75$ & 10300 & $0.05 \pm 0.02$ & $0.18 \pm 0.02$ & $0.37 \pm 0.01$ \\
$0.75-1.00$ & 2270 & $0.05 \pm 0.04$ & $0.24 \pm 0.04$ & $0.43 \pm 0.03$ \\
$1.00-1.25$ & 723 & $0.10 \pm 0.06$ & $0.30 \pm 0.06$ & $0.46 \pm 0.05$ \\
$1.25-1.50$ & 261 & $0.09 \pm 0.10$ & $0.31 \pm 0.10$ & $0.48 \pm 0.08$ \\
$1.50-2.00$ & 155 & $0.08 \pm 0.12$ & $0.35 \pm 0.13$ & $0.52 \pm 0.12$ \\
$>2.00$ & 42 & $0.16 \pm 0.21$ & $0.37 \pm 0.21$ & $0.49 \pm 0.28$ \\
\hline
\end{tabular}

Table 1: The Standard Model predictions for the number of accepted dilepton events, assuming $100 \%$ lepton identification for the used geometrical selection with an integrated luminosity of $100 \mathrm{fb}^{-1}$ and different mass bins. The expected asymmetries with their statistical errors for the different mass and rapidity intervals are also given.

It is interesting to compare the estimated accuracy of asymmetry measurements at the LHC with measurable quark asymmetries at a high energy linear $\mathrm{e}^{+} \mathrm{e}^{-}$collider. The most accurate and efficient asymmetry measurements with quarks in the final state can be obtained for the reaction $e^{+} e^{-} \rightarrow b \bar{b}$. To obtain asymmetry errors at $m_{\ell \ell} \approx 1 \mathrm{TeV}$ of about $2-3 \%$, at least 1000 accepted and tagged $\mathrm{b}-$ events are required. The cross section for the reaction $e^{+} e^{-} \rightarrow b \bar{b}$ at $1 \mathrm{TeV}$ center of mass energy is approximately $100 \mathrm{fb}$. Assuming an optimistic efficiency of about $10 \%$ to identify $\mathrm{b}$ events and measure correctly the charge of $\mathrm{b}$ flavoured jets, one can estimate the required LHC equivalent yearly luminosity to be about $100 \mathrm{fb}^{-1}$, corresponding to an average luminosity of $10^{34} \mathrm{sec}^{-1} \mathrm{~cm}^{-2}$. 


\section{$4 \quad \mathrm{~A}_{\mathrm{FB}}^{\ell}$ and Exotica}

We now discuss the sensitivity of the $\mathrm{A}_{\mathrm{FB}}^{\ell}$ measurements at the LHC with respect to two different exotic physics scenarios as implemented in PYTHIA. These are (a) composite models according to the model by E. Eichten et al. [11] and (b) a heavy $\mathrm{Z}^{\prime}$ with quark and lepton couplings identical to the Standard Model Z 15]. To demonstrate the sensitivity of asymmetry measurements two extreme scenarios for the total $Z^{\prime}$ width have been used. The width has been modified by changing the decay rate $\mathrm{Z}^{\prime} \rightarrow \mathrm{W}^{+} W^{-}$to very large values. For various other $\mathrm{Z}^{\prime}$ scenarios with their detailed lepton asymmetry predictions we refer to the literature [18]. The different model predictions for dilepton event rates are determined with the above selection criteria. For the asymmetries it is further required that the dilepton rapidities $|\mathrm{y}|$ are larger than 0.5 .

The compositeness scenario is simulated such that $\mathrm{u}$ and $\mathrm{d}$ quarks are composite with a scale $\Lambda^{ \pm}$, the sign indicates either a positive or a negative interference term. As a result of the contact interaction one expects a flattening of the steeply falling dilepton mass distribution as shown in Figure $3 \mathrm{a}$ for $\Lambda^{ \pm}$of $30 \mathrm{TeV}$ and for the Standard Model $\left(\Lambda^{ \pm}=\infty\right)$. Detailed discussions of these cross section changes can be found in [11]. In Table 2, dilepton event rates and asymmetries are given for different masses and $\Lambda^{ \pm}$scales. The central values for the different models have been obtained from a high statistic simulation equivalent to about 1000 $\mathrm{fb}^{-1}$. Taking dilepton cross section uncertainties of up to $20 \%$ into account, the event rate with

\begin{tabular}{|c|c|c|c|c|c|}
\hline mass $\left(\ell^{+} \ell^{-}\right)$ & \multicolumn{5}{|c|}{ number of events $\left(100 \mathrm{fb}^{-1}\right)$} \\
\hline$[\mathrm{TeV}]$ & $\Lambda^{\infty}$ & $\Lambda^{+}=20 \mathrm{TeV}$ & $\Lambda^{+}=30 \mathrm{TeV}$ & $\Lambda^{-}=20 \mathrm{TeV}$ & $\Lambda^{-}=30 \mathrm{TeV}$ \\
\hline $0.4-0.5$ & 14100 & 13940 & 14020 & 14380 & 14118 \\
$0.5-0.6$ & 6200 & 6127 & 6137 & 6462 & 6351 \\
$0.6-0.9$ & 5755 & 5591 & 5655 & 6180 & 5923 \\
$0.9-1.1$ & 978 & 885 & 940 & 1152 & 1023 \\
$1.1-1.4$ & 525 & 466 & 487 & 674 & 559 \\
$1.4-3.0$ & 267 & 320 & 257 & 558 & 353 \\
\hline & \multicolumn{5}{|c|}{$\mathrm{A}_{\mathrm{FB}}^{\ell}(|y|>0.5)$} \\
\hline $0.4-0.5$ & $0.283 \pm 0.010$ & 0.270 & 0.279 & 0.291 & 0.288 \\
$0.5-0.6$ & $0.320 \pm 0.015$ & 0.296 & 0.310 & 0.323 & 0.324 \\
$0.6-0.9$ & $0.345 \pm 0.015$ & 0.325 & 0.344 & 0.373 & 0.359 \\
$0.9-1.1$ & $0.392 \pm 0.035$ & 0.342 & 0.356 & 0.434 & 0.416 \\
$1.1-1.4$ & $0.421 \pm 0.048$ & 0.321 & 0.368 & 0.498 & 0.465 \\
$1.4-3.0$ & $0.451 \pm 0.068$ & 0.345 & 0.383 & 0.554 & 0.484 \\
\hline
\end{tabular}

Table 2: Accepted number of dilepton events and expected asymmetries for $|\mathrm{y}|$ larger than 0.5 for different compositeness scales $\Lambda^{ \pm}$, corresponding to an integrated luminosity of $100 \mathrm{fb}^{-1}$. For the Standard Model $\left(\Lambda^{\infty}\right)$ the statistical errors of the asymmetry results are also given.

masses above $1 \mathrm{TeV}$ alone will perhaps not be significant enough to observe compositeness with $\Lambda^{ \pm}$scales of about 25-30 TeV. However, the asymmetries, as shown in Figure $3 \mathrm{~b}$ and Table 2 will improve the sensitivity to compositeness phenomena considerable. The combination of cross section and asymmetry measurements should thus allow to increase the sensitivity at the 
two sigma level to $\Lambda^{ \pm}$values of almost $30 \mathrm{TeV}$.
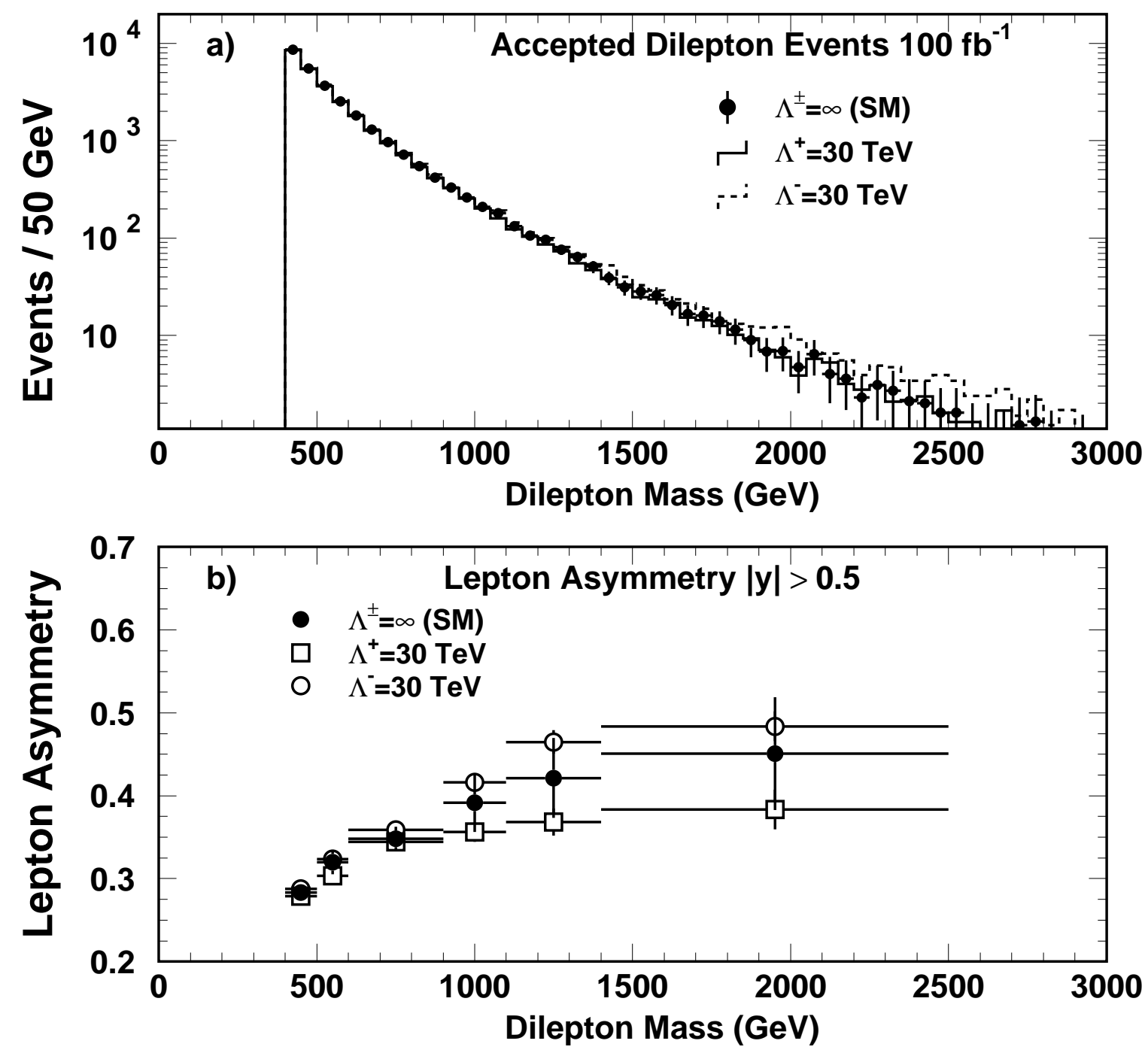

Figure 3: Expected dilepton mass distributions (a) and lepton asymmetries (b) for the Standard Model and for quark compositeness with different $\Lambda^{ \pm}$values.

The sensitivity of off- and on-resonance lepton asymmetry measurements to a $Z^{\prime}$ is demonstrated with two extreme scenarios. For this study a $\mathrm{Z}^{\prime}$ with a mass of $1 \mathrm{TeV}$ and a width of either $\approx 120 \mathrm{GeV}$ or of $\approx 860 \mathrm{GeV}$ are used. The resulting dilepton mass distributions from the exchange of photon, $Z$ and $Z^{\prime}$ are compared in Figure $4 a$ with the ones from the Standard Model with photon and $\mathrm{Z}$ exchange only. Clear mass peaks will demonstrate the presence of such a $\mathrm{Z}^{\prime}$ boson up to a few TeV. Only for a very broad $\mathrm{Z}^{\prime}$ the dilepton cross section measurement alone might not be sufficient. However, lepton asymmetries even far away from the $Z^{\prime}$ peak, as shown in Figures 4b, reveal large deviations from the Standard Model scenarios. We have also studied the continuum asymmetries for higher $\mathrm{Z}^{\prime}$ masses with relatively large width and 
find significant deviations from the Standard Model neutral current structure up to masses of about $2.5 \mathrm{TeV}$.
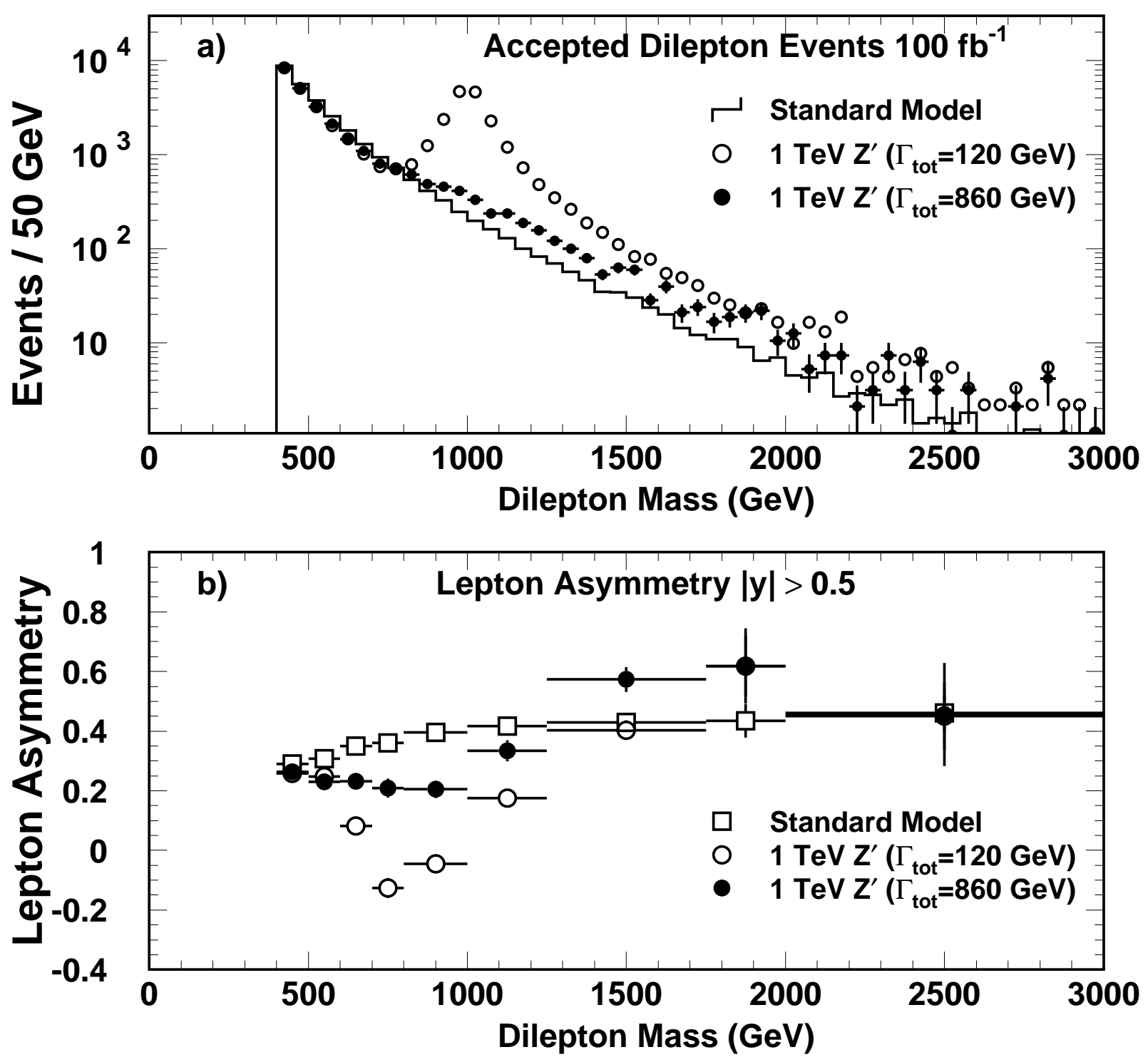

Figure 4: a) Expected dilepton mass distributions (a) and asymmetries (b) for the Standard Model and for two exotic $\mathrm{Z}^{\prime}$ scenarios. 


\section{Summary}

We have demonstrated the feasibility of lepton forward-backward asymmetry measurements with dilepton continuum events at the LHC. Such measurements, assuming the expected performance of ATLAS and CMS, appear to be almost straight forward. For an integrated lu-

minosity of $100 \mathrm{fb}^{-1}$, lepton asymmetry measurements will allow an accurate study of neutral current interference effects up to dilepton masses of about $2 \mathrm{TeV}$. The obtainable asymmetry accuracy is comparable with the ones at a $1 \mathrm{TeV}$ linear $\mathrm{e}^{+} \mathrm{e}^{-}$collider with quark final states and an integrated luminosity of about $100 \mathrm{fb}^{-1}$.

From a study of two different exotic scenarios, quark compositeness or exotic $\mathrm{Z}^{\prime}$ models, we conclude that continuum lepton asymmetries at the LHC can be considered as an additional and accurate experimental tool to observe and study new physics in the TeV center of mass energy domain.

\section{Acknowledgements}

I am grateful to E. Richter-Was for many detailed discussions about this analysis and her critical comments on the manuscript. I also would like to thank F. Pauss for several stimulating discussions and F. Behner for his suggestions on how to write an efficient fitting program.

\section{References}

[1] M. Davier, presentation at the 21. International Conference on High Energy Physics (1982), Journal de Physique colloque C3-471 eds. P. Petiau and M. Porneuf (Dec. 1982) and S. L. Wu, Phys. Rep. 107 (1984) 59 and further references therein.

[2] The LEP Collaborations, CERN/PPE/95-172 (24 November 1995).

[3] Discussions and further references on the use of forward-backward fermion asymmetries in high energy $\mathrm{e}^{+} \mathrm{e}^{-}$collisions can be found in:

G. Barbiellini et al., Physics at LEP, CERN 86-02 Vol II pages 46-54; A. Blondel et al., ECFA Workshop on LEP200, CERN87-08 and ECFA 87/108 Vol. II pages 414-452; and V. Angelopoulos et al., Physics at Future Accelerators, CERN 87-07 Vol. I pages 80-122.

[4] UA1 Collaboration, C. Albajar et al., Z. Phys. C44 (1989) 15; and CDF Collaboration, F. Abe et al., Phys. Rev. Lett. 67(1991) 1502. 
[5] P. Langacker et al., Phys. Rev. D30(1984) 1470;

for a recent review and further references therein see:

M. Cvetič and S. Godfrey, Electro-weak Symmetry Breaking and Beyond the Standard Model, eds T. Barklow, S. Dawson, H. Haber and J. Seigrist (World Scientific 1995).

[6] P. Camarri et al. LHC ECFA Workshop, CERN 90-10 and ECFA 90-133, Vol. II, 704.

[7] ATLAS Letter of Intent, CERN/LHCC/92-4 and LHCC/I2;

ATLAS Technical Proposal, CERN/LHCC/94-43 and LHCC/P2.

[8] CMS Letter of Intent, CERN/LHCC/92-3 and LHCC/I1;

Technical Proposal, CERN/LHCC/94-38 and LHCC/P1.

[9] P. Fisher et al., Phys. Lett. B356 (1995) 404.

[10] J. L. Rosner, Phys Rev. D35(1987) 2244.

[11] E. Eichten et al., Rev. Mod. Phys. 56 (1984) 579 and Rev. Mod. Phys. 58 (1985) 1065.

[12] J. L. Rosner, FERMILAB-PUB-95/394-T submitted to Phys. Rev. D.

[13] T. Sjöstrand CERN-TH.7112/93; Comp. Phys. Comm. 39 (1986) 347 and T. Sjöstrand and M. Bengtsson, Comp. Phys. Comm. 43 (1987) 43.

[14] The PYTHIA subprocesses 1 and 165 are used to simulate dilepton events according to the formalism given in [11].

[15] The PYTHIA subprocess 141 is used to simulate $\mathrm{Z}^{\prime}$ production according to the formalism given in G. Altarelli et al., Z. Phys. C45 (1989) 109.

[16] The CTEQ2 structure functions (mainly CTEQ2L, the PYTHIA 5.7 default) have been used for the simulation.

[17] ATLAS TP [7] page 228 and D. Denegri private communication.

[18] For a recent review of asymmetries and $\mathrm{Z}^{\prime}$ Models see for example [12] and further references therein. 